\title{
Purinergic Signals Regulate Daily S-Phase Cell Activity in the Ciliary Marginal Zone of the Zebrafish Retina
}

\author{
Maria Jimena Ricatti, ${ }^{*}$ Ariadna Gabriela Battista, ${ }^{*}$ Maria Zorrilla Zubilete, ${ }^{\ddagger}$ \\ and Maria Paula Faillace ${ }^{1 *+}$ \\ ${ }^{*}$ Facultad de Medicina, Universidad de Buenos Aires, Ciudad de Buenos Aires, Argentina, \\ ${ }^{+}$Instituto de Química y Fisicoquímica Biológicas (IQUIFIB), Consejo Nacional de Investigaciones \\ Científicas y Técnicas (CONICET), Ciudad de Buenos Aires, and ${ }^{\ddagger}$ Centro de Estudios \\ Farmacológicos y Botánicos (CEFYBO-CONICET), Argentina
}

\begin{abstract}
Regeneration and growth that occur in the adult teleost retina have been helpful in identifying molecular and cellular mechanisms underlying cell proliferation and differentiation. Here, it is reported that S-phase cell number, in the ciliary marginal zone (CMZ) of the adult zebrafish retina, exhibits daynight variations with a mid-light phase peak. Oscillations persist for $24 \mathrm{~h}$ in constant darkness (DD), suggesting control by a circadian component. However, variations in the S-phase nuclei number were rapidly dampened and not present during and after a second day in DD. An ADP $\beta S$ treatment significantly enhanced S-phase activity at night to mid-light levels, as assessed by in vivo BrdU incorporation in a 2-h interval. Moreover, daylight increase in S-phase cell number was completely abolished when extracellular nucleotide levels or their extracellular hydrolysis by ectonucleoside triphosphate diphosphohydrolases (NTPDases) were significantly disrupted or when a selective antagonist of purinergic P2Y1 receptors was intraocularly injected before BrdU exposure. Extracellular nucleotides and NTPDase action were also important for maintaining nocturnal low levels of S-phase activity in the CMZ. Finally, we showed that mRNAs of NTPDases 1, 2 (3 isoforms), and 3 as well as of P2Y1 receptor are present in the neural retina of zebrafish. NTPDase mRNA expression exhibited a 2-fold increment in light versus dark conditions as assessed by quantitative RT-PCR, whereas P2Y1 receptor mRNA levels did not show significant day-night variations. This study demonstrates a key role for nucleotides, principally ADP as a paracrine signal, as well as for NTPDases, the plasma membrane-bound enzymes that control extracellular nucleotide concentration, for inducing S-phase cell entry in the CMZ-normally associated with retinal growth—throughout the light-dark cycle.
\end{abstract}

Key words zebrafish retina, ciliary marginal zone, extracellular nucleotides, S-phase cell rhythm

1. To whom all correspondence should be addressed: Maria Paula Faillace, Laboratorio de Neurociencias, Departamento de Fisiología, Facultad de Medicina, Paraguay $21557^{\circ}$ piso, C1121ABG Ciudad Autónoma de Buenos Aires, Argentina; e-mail: pfaillace@qb.ffyb.uba.ar.

JOURNAL OF BIOLOGICAL RHYTHMS, Vol. 26 No. 2, April 2011 107-117

DOI: $10.1177 / 0748730410395528$

(C) 2011 SAGE Publications 
Eyes in teleost fish grow throughout life, and the mature retina matches this growth by stretching and the continuous genesis of new cells (Johns and Fernald, 1981; Stenkamp, 2007). Cell proliferation and differentiation occur in a neuroepithelial zone at the interface between neural retina and ciliary epithelium, called the ciliary marginal zone (CMZ) (Lyall, 1957; Reh and Fischer, 2001). Within mature retina, rod photoreceptors differentiate from precursor cells within the outer nuclear layer (ONL) (Johns and Fernald, 1981). In embryonic development, retinal cells clonally originate from a common cell precursor. However, whether a unique cell precursor exists in the CMZ and the nature of the extrinsic and intrinsic signals that regulate retinogenesis are subjects of intense investigation. Light, for a photosensitive tissue such as the retina, might regulate normal growth and cell proliferation like in other zebrafish larval tissues (Dekens et al., 2003). In fact, daily variations of rod precursor proliferation during the light-dark (LD) cycle has been described in a teleost fish (Chiu et al., 1995). Several studies in the vertebrate retina have revealed that a circadian clock drives many retinal functions (Cahill and Besharse, 1995).

Extracellular nucleotides/nucleosides (eNCT/S) in the retina are important cues for neuron-glia communication (Newman, 2006). ATP is released by exocytosis in the nervous system (Burnstock, 2006) and via gap junction hemi-channels in the retina (Pearson et al., 2005). Di- and triphosphate nucleotides such as ATP, ADP, and UTP are extracellularly dephosphorylated by ectonucleoside triphosphate diphosphohydrolases (ENTPDases). The coordinated action of ENTPDases and an ecto-5'-nucleotidase dephosphorylates extracellular ATP to adenosine (Hunsucker et al., 2005). eNCT/S act via specific purinergic or $P$ receptors in the plasma membrane (Burnstock, 1978; 2007). Studies on retinal development in the chick embryo suggest a role for eNCT/S in regulating progenitor cell proliferation (Pearson et al., 2005; França et al., 2007). Interestingly, NTPDase 2 and ADP participate in eye and retina morphogenesis in Xenopus laevis (Massé et al., 2007).

Therefore, we investigated the role of extracellular ATP and its metabolites in regulating S-phase cell activity in the CMZ of the zebrafish retina, which we have found follows day-night oscillations during the LD cycle that persist for $24 \mathrm{~h}$ in constant darkness (DD). The number of S-phase nuclei was highest at the mid-light phase and mid-subjective day. S-phase cell number was regulated by eNCT in both light and darkness. mRNAs of NTPDases 1, 2, and 3 are expressed in the zebrafish retina, and their levels showed significant day-night variations.

\section{MATERIALS AND METHODS}

\section{Materials}

Materials included paraformaldehyde; PBS (137 mM $\mathrm{NaCl}, 2.7 \mathrm{mM} \mathrm{KCl}, 5.7 \mathrm{mM}$ phosphate, $\mathrm{pH}: 7.4)$; Tween-20; MS-222; sucrose; apyrase (EC-3.6.1.5); hexokinase (EC-2.7.1.1); 5-bromo-2'-deoxyuridine

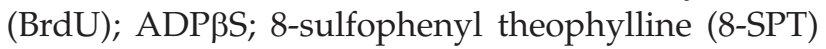
(Sigma, St Louis, MO); MRS2211, MRS2179, NF110, NF157, and ARL67156 trisodium salt (Tocris, Bristol, UK); mouse monoclonal anti-BrdU antibody (Roche, Mannheim, Germany); goat anti-mouse ALEXA 488 antibody, random primers, dNTPs, MMLV, DTT, RNAseOUT, and sense and antisense primers (Invitrogen, Carlsbad, CA); Total RNA Extraction Kit (RBC Biosciences, Taiwan); GoTaq-DNA polymerase, dNTPs-mix, DNasa-I (Promega, Madison, WI); realtime PCR mix (Biodynamics, Buenos Aires, Argentina); and ultra pure agarose (GenBiotech, Buenos Aires, Argentina).

\section{Animals}

Adult zebrafish (Danio rerio) of about $3 \mathrm{~cm}$ and $1.5 \mathrm{~g}$ were maintained at $28.5{ }^{\circ} \mathrm{C}$ in a $14: 10 \mathrm{~h}$ LD cycle (lights on $0700 \mathrm{~h}$ and off $2100 \mathrm{~h}$ ) and fed twice a day. A group of zebrafish, used for night intervals, was raised in an inverted phase (I) 14:10 h LD cycle (lights on $2000 \mathrm{~h}$ and off $1000 \mathrm{~h}$ ) in a separate room. Other groups of zebrafish were transferred to DD. Animals were euthanized by immersion in MS-222 anesthetic solution $(0.02 \% \mathrm{wt} / \mathrm{vol})$ and then decapitated and enucleated, at 4 different intervals defined as Zeitgeber time (ZT2, ZT9, ZT16, and ZT21; ZT0 indicates lights on and ZT14 lights off) during the LD cycle or first, second, and fourth days in DD. A group of zebrafish from the (I) LD cycle were euthanized at ZT9 (i.e., mid-light for this group). The Committee on Animal Research at University of Buenos Aires approved protocols for animal use and care.

\section{Intravitreal Injections}

5-Bromo-2'-deoxyuridine administration. BrdU, a thymidine analogue, incorporates into DNA during the 
S-phase of the cell cycle. Anesthetized zebrafish were intraocularly injected with $0.5 \mu \mathrm{L}$ of a sterile saline $(0.65 \%)$ solution containing $20 \mu \mathrm{g} / \mu \mathrm{L}$ of $\mathrm{BrdU}, 2 \mathrm{~h}$ before euthanasia. BrdU was injected in control or previously treated eyes after $20 \mathrm{~h}$ of each treatment. Zebrafish in darkness were injected and euthanized in dim far red light.

eNCT hydrolyzing enzyme treatment. Apyrase dephosphorylates diphosphate and triphosphate nucleotides whereas hexokinase hydrolyzes ATP to phosphorylated D-hexose plus ADP. We injected $0.5 \mu \mathrm{L}$ of $25 \mathrm{U} /$ $\mathrm{mL}$ apyrase, hexokinase, or heat-inactivated enzymes (boiled for $5 \mathrm{~min}$ ) once. Zebrafish were euthanized 22 $\mathrm{h}$ later to prepare eyecups. Apyrase or hexokinase remains active within the vitreous at least for $20 \mathrm{~h}$ (Battista et al., 2009).

Ecto-ATPase activity inhibitor treatment. ARL67156, an ATP analogue, selectively inhibits ecto-ATPase activity without activating purinergic receptors (Robson et al., 2006). We injected $100 \mu \mathrm{M}$ ARL67156 (Westfall et al., 1997; Newman, 2003) via the intravitreal route in $0.5 \mu \mathrm{L}$ of saline solution $\left(\mathrm{pIC}_{50}=4.62\right.$ and 5.1 in human blood and rat vas deferens, respectively). Zebrafish were euthanized $22 \mathrm{~h}$ later.

eNCT/S agonist and antagonist treatment. We injected $5 \mu \mathrm{M}$ MRS2211, $1 \mu \mathrm{M}$ MRS2179, $10 \mu \mathrm{M}$ 8-SPT, or $5 \mu \mathrm{M}$ NF157 plus $5 \mu \mathrm{M}$ NF110, and $22 \mathrm{~h}$ later zebrafish were euthanized (after $2 \mathrm{~h}$ of BrdU exposure). Additionally, a group of zebrafish were injected with $25 \mu \mathrm{M}$ ADP $\beta S$, a slightly hydrolysable ADP analogue. Control eyes from different zebrafish were injected with sterile saline.

MRS2211. P2Y13 receptor antagonist $\left(\mathrm{IC}_{50}=1.07 \mu \mathrm{M}\right)$ displays greater than 20-fold selectivity over P2Y1. Agonist relative specificity: ADP>>ATP. MRS2179: P2Y1 receptor antagonist $\left(\mathrm{IC}_{50}=0.330 \mu \mathrm{M} ; \mathrm{K}_{\mathrm{i}}=100 \mathrm{nM}\right)$; selective over $\mathrm{P} 2 \mathrm{X} 1 / \mathrm{X} 3 / \mathrm{X} 2 / \mathrm{X} 4$ and $\mathrm{P} 2 \mathrm{Y} 2 / \mathrm{Y} 4 / \mathrm{Y} 6$ receptors. Agonist relative specificity: $A D P \beta S>$ ADP $>>$ ATP (Burnstock, 2007). NF110: high-affinity $\mathrm{P} 2 \mathrm{X} 3$ receptor antagonist $\left(\mathrm{IC}_{50}=0.527 \mu \mathrm{M} ; \mathrm{K}_{\mathrm{i}}=\right.$ $36 / 82 / 4144 \mathrm{nM}$ for ATP-activated $\mathrm{P} 2 \mathrm{X} 3 / \mathrm{X} 1 / \mathrm{X} 2$, respectively); shows no activity on $\mathrm{P} 2 \mathrm{Y} 1 / \mathrm{Y} 2 / \mathrm{Y} 11$ receptors $\left(\mathrm{IC}_{50}>10 \mu \mathrm{M}\right)$. NF157: Potent antagonist of P2Y11 receptors $\left(\mathrm{IC}_{50}=0.463 \mu \mathrm{M}\right)$; selective for ATPactivated P2Y11 and P2X1 over P2Y1/Y2, P2X2/ $\mathrm{X} 3 / \mathrm{X} 4 / \mathrm{X} 7$ receptors. Agonist relative specificity: ATP $\gamma$ S $>$ ATP (Burnstock, 2007). 8-sulfophenyl theophylline (8-SPT): adenosine P1 receptors antagonist $\left(\mathrm{K}_{\mathrm{i}}=1.2 \mu \mathrm{M}\right)$.

\section{Immunocytochemistry}

To prepare eyecups, the cornea, lens, and vitreous were removed. Eyecups were fixed with $4 \%$ paraformaldehyde for $1 \mathrm{~h}$ at room temperature (RT); incubated in 5\%,10\% (30 min), and 20\% sucrose (overnight); and embedded in tissue freezing medium. We collected $16-\mu \mathrm{m}$ cryosections onto gelatin-subbed slides, which were kept at $-20^{\circ} \mathrm{C}$. Retinal sections were incubated in $2 \mathrm{M} \mathrm{HCl}$ for $15 \mathrm{~min}$ at $37^{\circ} \mathrm{C}$ and thoroughly washed with PBST (PBS plus 0.1\% Tween-20). Then, sections were incubated $1 \mathrm{~h}$ at RT in 5\% normal goat serum (NGS) and overnight with an antibody against BrdU (1:200 in 3\% NGS) at $4{ }^{\circ} \mathrm{C}$. Sections were washed and incubated in darkness for $2 \mathrm{~h}$ at RT, with a fluorescent antibody (1:400 in 3\% NGS). Omission of antibodies was performed as negative controls.

\section{Counting of BrdU-Positive Nuclei}

Eyecups were cut in the sagittal plane (nasal to temporal). Each slide contained sections from different portions of the eyecup. We identified BrdU-positive nuclei focusing through the section in both $\mathrm{CMZ}$ under microscopic observation. We counted all BrdUpositive nuclei in 10 to 12 nonadjacent sections from each eye. Nuclei in the nonlaminated retina at both ends were considered CMZ nuclei. Differences among groups were analyzed using 1-way ANOVA, followed by Tukey-Kramer multiple comparison test or Student $t$ test.

\section{Microscopy}

Counting was performed with an epifluorescence microscope (Olympus BX50, Japan; objective 40X-NA: 0.75). Confocal images were captured with an FV1000 Fluoview spectral microscope (Olympus; objective SAPO-60X oil-NA: 1.35). Two-dimensional confocal and differential interference contrast (DIC) images were obtained from Z-stacks by using Fluoview software.

\section{Reverse Transcription (RT) and Polymerase Chain Reaction (PCR)}

Groups of 5 zebrafish were euthanized at ZT7-9 (mid-light phase) in bright light and ZT14-16 (1:30 h of darkness) in dim far red light. Ten neural retinas were homogenized in a buffer of a RNA purification kit and considered a sample. Three to 4 sample replicates 
Table 1. Specific primers for each target gene and reference gene ( $\beta$-actin).

\begin{tabular}{lll}
\hline Gene & \multicolumn{1}{c}{ Sense } & \multicolumn{1}{c}{ Antisense } \\
\hline NTPDase 1 & TCACAGACACAGCGAAACCC & GAATGTGCAACGGAGGTAAGC \\
NTPDase $\mathbf{m q}$ & GAGCAGCAGCACGTAGCC & GACCTCAGCCGACTCTTTGG \\
NTPDase $\mathbf{m g}$ & CTTACGCCTCTTTGACACG & AGTCAGATGGATAGCAGGGATG \\
NTPDase2 $\mathbf{m v}$ & TGATGGAGTCTTTCAGCCCAAC & AGCAGCGTCCTCTAAATGAGC \\
NTPDase3 & GGCTGGATCACTGTCAACTACC & CCCAAATCCAATGACCCAACTG \\
$\mathbf{P 2} \mathbf{1}$ receptor & TATGGACAATGCTCCGCTTAGG & CGCTCTCATGTTCAGGTTCTTC \\
$\boldsymbol{\beta}$-actin & TCCCAAAGCCAACAGAGAGAAG & GTCACACCATCACCAGAGTCC \\
\hline
\end{tabular}

Primers were selected by using exon sequences from zebrafish genome (Ensembl database). Selected primer pairs did not hybridize with other NTPDase enzyme or isoform $(m q, m g, m v)$.

\section{Quantitative PCR Data Analysis}

Analysis was performed throughout the $2^{-\Delta \Delta C T}$ method as described by Livak and Schmittgen (2001). An arbitrary fluorescence threshold of 0.06 was used to obtain $\mathrm{C}_{\mathrm{T}}$ values from each curve. We chose cDNA samples from mid-light intervals (ZT7-9) as the interest sample and beginning of darkness (ZT14-16) as the calibrator sample. Both $C_{T}$ values were previously normalized with $\beta$-actin. We obtained a ratio that indicates fold change of mRNA levels of each target gene at ZT7-9 over ZT14-16. For the calibrator sample, $2^{-\Delta \Delta C T}$ value equals 1 . Relative values from each pool of 10 retinas are reported. Standard deviations (SD) from triplicates were propagated by using standard methods and confidence

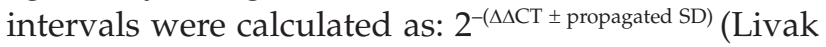
and Schmittgen, 2001).

\section{RESULTS}

\section{S-Phase Nuclei Number in the CMZ Shows Day-Night Variations That Persist for $24 \mathrm{~h}$ in DD}

We counted BrdU-positive nuclei (S-phase nuclei) within the following 2-h intervals: ZT0-2 and ZT7-9 in light, ZT14-16 and ZT19-21 in darkness (Figure 1A). Despite the scant number of BrdU-positive nuclei per $\mathrm{CMZ}$ /section, this parameter showed daily variations with a peak at ZT7-9, that is, after 7 to $9 \mathrm{~h}$ of light (4.78 \pm 0.98 nuclei, $n=8$ zebrafish). After $2 \mathrm{~h}$ of darkness (ZT14-16), S-phase cell number decreased to a minimum (1.49 \pm 0.30 nuclei, $n=5$ zebrafish), which was significantly different from BrdU-positive nuclei after $7 \mathrm{~h}$ of darkness (ZT19-21: $2.80 \pm 0.56$ nuclei, $n=$ 8 zebrafish). At dawn, ZT0-2, BrdU-positive nuclei were (2.34 $\pm 0.78, n=7$ zebrafish) not different from night values. The ratio of S-phase nuclei at the midlight peak to the trough at night was 3.2 to 1 . Upon transfer to DD, S-phase cell number showed significant variations among intervals for $24 \mathrm{~h}$ (ZT0-2: $2.72 \pm$ 0.44; ZT7-9, 4.83 \pm 0.43; ZT14-16, $1.05 \pm 0.07$; ZT19-21, $1.80 \pm 0.32 ; n=4$ zebrafish per interval; Figure 1B) with a maximum to minimum ratio of 4.6 to 1 . Subjective 

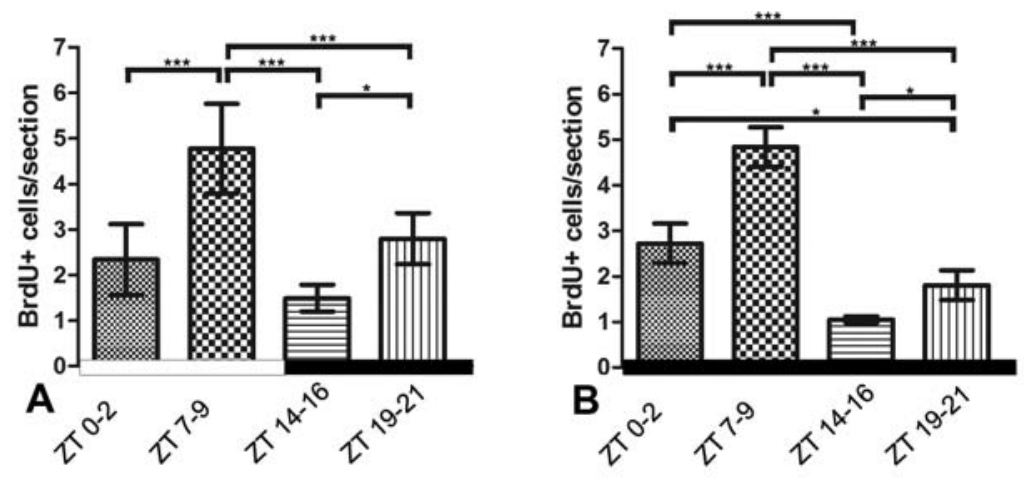

was significantly higher at ZT14-16 and ZT19-21 (Figure 1D) than at ZT14-16 in LD (Figure 1A) $(p<0.05$, Tukey-Kramer after ANOVA). Data from day 4 in DD intervals (except for ZT7-9) were significantly higher than those observed during the second day in DD $(p<0.05$, $p<0.01$; Figure $1 C$ vs. 1D).

Supplementary Figure S2 depicts S-phase nuclei number in the CMZ of 2 groups of zebrafish injected with BrdU at ZT7 and euthanized at ZT9 (mid-light
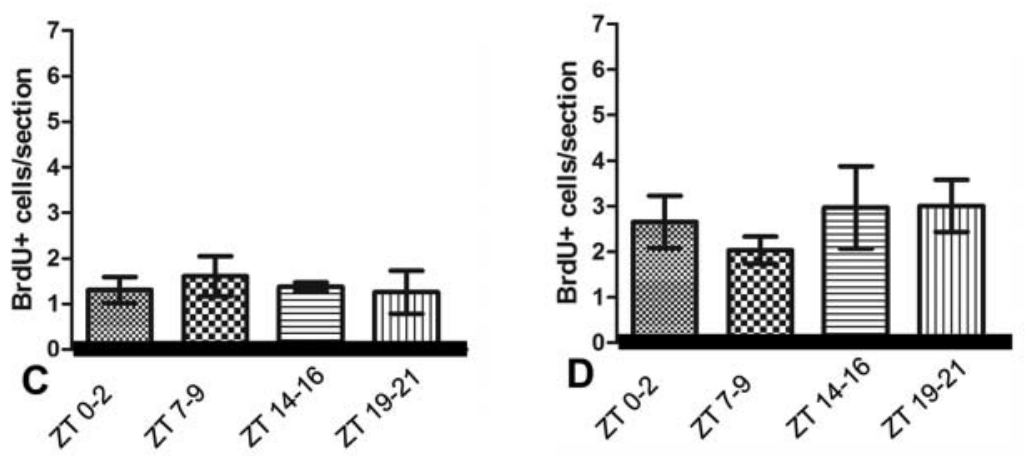

Figure 1. Number of BrdU-positive nuclei plotted as a function of time of day (ZT) in a 14:10 h LD cycle (A) or during the first (B), second (C), or fourth (D) day in DD. Zebrafish were handled either in light or under far red illumination. Values are BrdU-positive cell number per ciliary marginal zone (CMZ) per retinal section and are mean \pm SD $(n=4-8$ zebrafish per ZT interval; ZT0 is lights on and ZT14 lights off). Brackets indicate significant differences between intervals. ${ }^{* *} p<0.001$; ${ }^{*} p<0.05$, Tukey-Kramer multiple comparison test after 1-way ANOVA.

day values were significantly higher than values at subjective night. Confocal images with a representative S-phase nuclei number at ZT7-9 and ZT14-16 in LD and 1 day in DD are shown in Supplementary Figure S1A-D and E-H, respectively. In contrast, BrdU-positive nuclei number during the second day in DD (ZT0-2: $1.30 \pm 0.29$; ZT7-9: $1.60 \pm 0.43$; ZT1416: $1.37 \pm 0.09 ;$ ZT19-21: $1.26 \pm 0.47, n=4$ zebrafish per interval; Figure 1C) or the fourth day in DD (ZT0-2: $2.65 \pm 0.57$; ZT7-9: $2.03 \pm 0.30$; ZT14-16: $2.97 \pm$ 0.90 ; ZT19-21: $3.00 \pm 0.57, n=6$ zebrafish per interval; Figure 1D) did not show significant variations among intervals. During subjective days 2 and 4, BrdUpositive nuclei failed to increase to the LD mid-light peak (Figure 1A) versus DD through all ZT intervals (Figure 1C and D; $p<0.001$, Tukey-Kramer after ANOVA). Moreover, during the second day in DD, values were not statistically different from the night minimum in LD (Figure 1A vs. 1C). In contrast, BrdU-positive nuclei number during day 4 in DD for both groups of fish). One of the groups was kept in a different room in a 12-h shifted phase relative to the other. BrdU-positive nuclei number in both groups did not show significant differences (ZT7-9: $4.78 \pm 0.98, n=8$; ZT7-9(I): $4.72 \pm 0.15 n=3$ zebrafish).

\section{Effect of eNCT Hydrolyzing Enzymes and an EctoATPase Selective Blocker on S-Phase Cell Activity During the LD Cycle}

Figure 2 shows the effect of apyrase or hexokinase activity on S-phase nuclei number at different intervals. Apyrase treatment significantly decreased BrdUpositive nuclei number at the $\mathrm{CMZ}$ at both night and daylight as compared to heat-inactivated apyrase-treated retinas (ZT7-9: $3.99 \pm 0.26, n=3$; ZT14-16: $1.69 \pm 0.30, n=3$ ) and saline controls (ZT79: $4.20 \pm 0.26, n=3$; ZT14-16: $1.67 \pm 0.10, n=3)$. Control data were not significantly different (ZT7-9: $p=0.3788$ and ZT14-16: $p=0.9864$ ), and they were pooled in Figures 2A and B. At ZT7-9, S-phase cell number in apyrase-treated retinas diminished below control night levels (apyrase ZT7-9: $0.61 \pm 0.20, n=4$ [Figure 2A] vs. control ZT14-16: $1.68 \pm 0.20, n=6$ [Figure 2B] and ZT19-21: $2.19 \pm 0.34, n=3$ [Figure $2 \mathrm{C}] ; p<0.001)$. The low number of BrdU-positive nuclei at night was further decreased to either 1 or zero in the majority of the quantified sections (ZT1416: apyrase $0.60 \pm 0.15, n=4$ vs. control $1.68 \pm 0.20$, $n=6$ [Figure 2B]; ZT19-21: apyrase $0.39 \pm 0.12, n=4$ vs. control $2.19 \pm 0.34, n=3$ [Figure 2C]). In contrast, intravitreal activity of hexokinase showed no significant effect on S-phase nuclei number either in light or dark conditions as compared to the heat-inactivated 

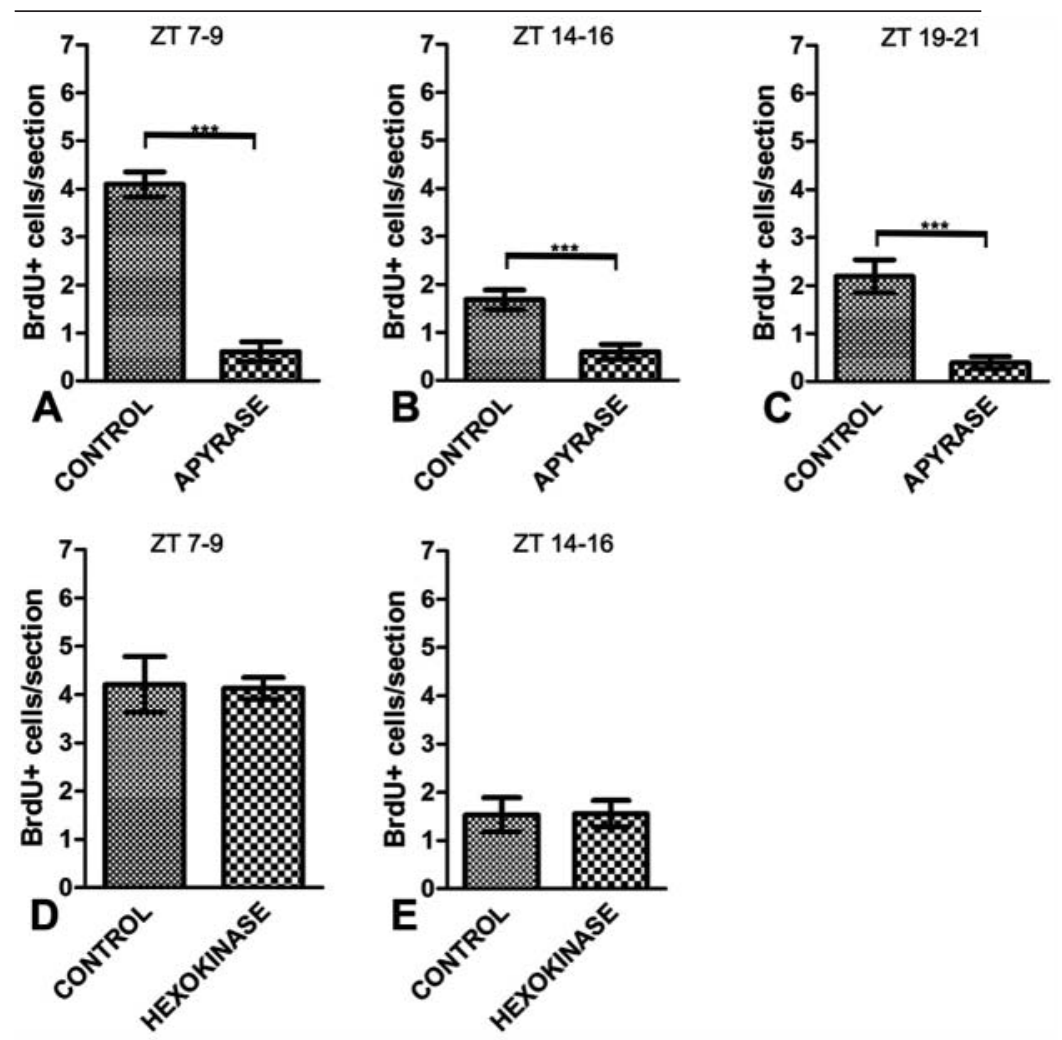

Figure 2. Apyrase or hexokinase activity effect on BrdU-positive nuclei number analyzed at daily intervals during a 14:10-h LD cycle. Eyes underwent intravitreal injection of saline, apyrase, or heat-inactivated apyrase (A-C) or hexokinase or heat-inactivated hexokinase (D, E). Twenty hours later, they were injected with a solution containing BrdU $(20 \mu \mathrm{g} / \mu \mathrm{L})$. Zebrafish were euthanized $2 \mathrm{~h}$ later at the end of the indicated ZT interval (ZT0 is lights on and ZT14 lights off). Values represent the number of BrdU-positive cells per ciliary marginal zone (CMZ)/retinal section and are mean \pm SD. Controls: ZT7-9 and ZT14-16 saline plus heat-inactivated apyrase ( $n=6$ zebrafish per ZT); ZT19-21 heat-inactivated apyrase ( $n=3$ zebrafish); ZT7-9 and ZT14-16 heat-inactivated hexokinase ( $n=4-5$ zebrafish). Apyrase- or hexokinase-treated retinas: $n=4$ zebrafish per ZT interval. Brackets indicate significant differences between treatments. ${ }^{* * *} p<0.001$ by Student $t$ test.

of S-phase cells in the CMZ at ZT7-9 in absence (I, J) or presence (K, L) of active apyrase and without $(\mathrm{M}, \mathrm{N})$ or with ARL67156 treatment $(\mathrm{O}, \mathrm{P})$.

\section{Specific eNCT Regulate \\ S-Phase Cell Activity in the CMZ During the LD Cycle}

Figure 3A-C depicts data showing that MRS2179 - a selective antagonist of ADP-activated P2Y1 metabotropic receptors-significantly reduced BrdUpositive nuclei number at mid-light, to levels below control values recorded at night (MRS2179: ZT7-9, $0.76 \pm 0.34, n=$ 4 [Figure 3A] vs. control: ZT14-16, $1.68 \pm$ $0.20, n=6$ [Figure 3B] and ZT19-21, 2.12 $\pm 0.28, n=5$ [Fig 3C]; $p<0.001$ ). MRS2179, like apyrase or ARL67156 treatments, significantly decreased BrdU-positive nuclei counts at night to less than a dividing nucleus in a 2-h window per retinal section (MRS2179: ZT14-16, 0.51 \pm 0.17 [Figure 3B] and ZT19-21, $0.60 \pm$ 0.39 [Figure 3C] $n=4$ zebrafish per group). In contrast, P2 receptor antagonists such as MRS2211, which blocks ADP action on P2Y13 receptors, showed no significant effect (MRS2211: ZT7-9, $3.95 \pm 0.21$ [Figure 3D]; ZT14-16, $1.97 \pm$ 0.10 [Figure 3E], $n=4$ zebrafish per group). Antagonists for ionotropic P2X1/2/3 and metabotropic P2Y11 receptors also failed in demonstrating

hexokinase-treated retinas (ZT7-9: hexokinase $4.13 \pm$ 0.22 and control $4.21 \pm 0.58, n=4$; ZT14-16: hexokinase $1.56 \pm 0.27, n=4$ and control $1.53 \pm 0.36, n=5$ ) (Figure 2D, E). Furthermore, when ARL67156known to inhibit NTPDase activity at the concentration tested-was injected within the vitreous (Figure 3 A-C), it significantly decreased BrdU-positive nuclei number at mid-light to levels below those normally observed at night (ARL67156: ZT7-9, $0.91 \pm 0.22, n=$ 4 [Figure 3A] vs. control: ZT14-16, $1.68 \pm 0.20, n=6$ [Figure 3B] and ZT19-21, $2.12 \pm 0.28, n=5$ [Figure 3C]; $p<0.001)$. ARL67156 treatment also decreased BrdU-positive nuclei number at night (ARL67156: ZT14-16, $0.29 \pm 0.05, n=4$ [Figure 3B]; ZT19-21, 0.32 $\pm 0.11, n=4$ [Figure 3C]). Supplementary Figure S1 shows confocal images with a representative number an ATP-induced effect on S-phase cell activity (NF110/157: ZT7-9, $3.97 \pm 0.07$ [Figure 3E]; ZT14-16, $1.73 \pm 0.13$ [Figure 3D]; $n=4$ zebrafish per group). Likewise, 8-SPT an antagonist for adenosine-activated $\mathrm{P} 1$ receptors did not alter BrdU-positive nuclei number at any interval examined (8-SPT: ZT7-9, 3.87 \pm 0.64 [Figure 3E]; ZT14-16, $1.94 \pm 0.32$ [Figure 3D]; $n=4$ zebrafish per group). For MRS2211, NF110/157 and 8-SPT control values were ZT7-9, $4.18 \pm 0.55$; ZT14-16, $1.68 \pm 0.25, n=4$ zebrafish per group. Consistent with this, 1 intravitreal injection of ADP $\beta S$, an agonist for P2Y1 receptors, caused a significant increase of the number of BrdU-positive nuclei in the CMZ at night (control: ZT14-16, $1.63 \pm$ $0.22, n=4$ [Figure 3F]). S-phase nuclei number in the presence of ADP $\beta S$ at ZT14-16 was statistically 

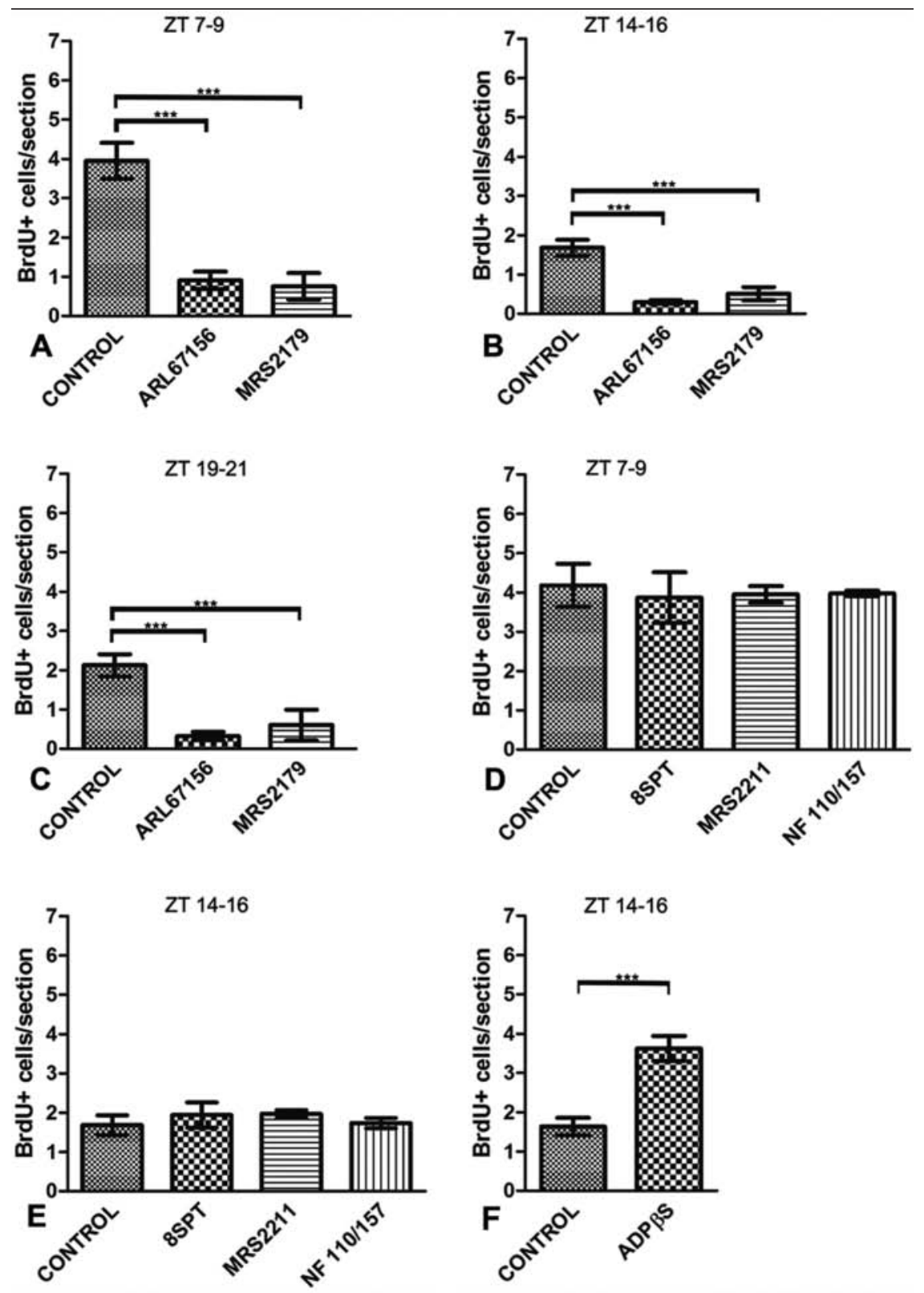

Figure 3. P receptor agonist and antagonists and an ectoATPase activity inhibitor effect on BrdU-positive nuclei number at different intervals during a 14:10-h LD cycle. Eyes underwent intravitreal injection with saline, ARL67156, or MRS2179 (A-C); saline, 8-SPT, MRS2211, or NF110/157 (D, E); or saline or ADP $\beta S$ (F). Twenty hours later, they were injected with a solution containing BrdU $(20 \mu \mathrm{g} / \mu \mathrm{L})$. After $2 \mathrm{~h}$, zebrafish were euthanized at the end of the indicated ZT interval (ZT0 is lights on and ZT14 lights off). Values represent BrdU-positive cell number per ciliary marginal zone $(\mathrm{CMZ}) /$ retinal section and are mean \pm SD. (A) ZT7-9: $n=4-7$ zebrafish. (B) ZT14-16: $n=4-6$ zebrafish. (C) ZT19-21: $n=4-5$ zebrafish. (D) ZT7-9 and (E) ZT14-16: $n=4$ zebrafish per group. (F) ZT14-16: $n=4-5$ zebrafish. Brackets indicate significant differences between treatments. ${ }^{* * *} p<0.001$, as assessed by Tukey-Kramer multiple comparison test after 1-way ANOVA or Student $t$ test.

indistinguishable from mid-light values in absence of exogenous nucleotides (ADP $\beta S:$ ZT14-16, $3.62 \pm$ $0.32, n=5$ [Figure 3F] vs. control: ZT7-9, $3.95 \pm 0.45$ $n=7$ [Figure 3A]; $p=0.1996$ ). Supplemen-tary Figure S1 depicts 2-dimensional confocal images with a representative $S$-phase nuclei number in the CMZ at ZT14-16 in absence $(Q, R)$ or presence $(S, T)$ of ADP $\beta$.

\section{NTPDase mRNA Levels Show Day-Night Variations in the Zebrafish Retina}

RT-PCR experiments demonstrated mRNA expression of NTPDases 1, 2, and 3 as well as of ADP-activated P2Y1 metabotropic receptor in the zebrafish neural retina (Figure 4C). One band was observed with each primer pair, and amplicon lengths matched expected molecular weights. Furthermore, $m q, m v$, and $m g$ isoforms for NTPDase2 previously described in other tissues were identified. To reliably detect $m g$ isoform, we used $2 \mu \mathrm{g}$ of cDNA, whereas $1 \mu \mathrm{g}$ was sufficient to identify $m v$ and $m q$ isoforms or NTPDases 1 and 3 . NTPDase mRNA levels during the LD cycle showed a higher expression in mid-light phase (ZT7-9) than at the beginning of night, after 1:30 h of darkness (ZT14-16) (Figure 4A, B). Expression level of NTPDase mRNAs between intervals was on average about 2- to 3-fold. In contrast, P2Y1 receptor mRNA levels did not show apparent day-night variations.

\section{DISCUSSION}

Here we report daily variations in S-phase progenitor cell activity in the CMZ of the adult zebrafish retina, with highest values at the mid-light phase. The observed day-night oscillations persist for $24 \mathrm{~h}$ in free-running conditions. S-phase activity in the CMZ is strongly regulated by 


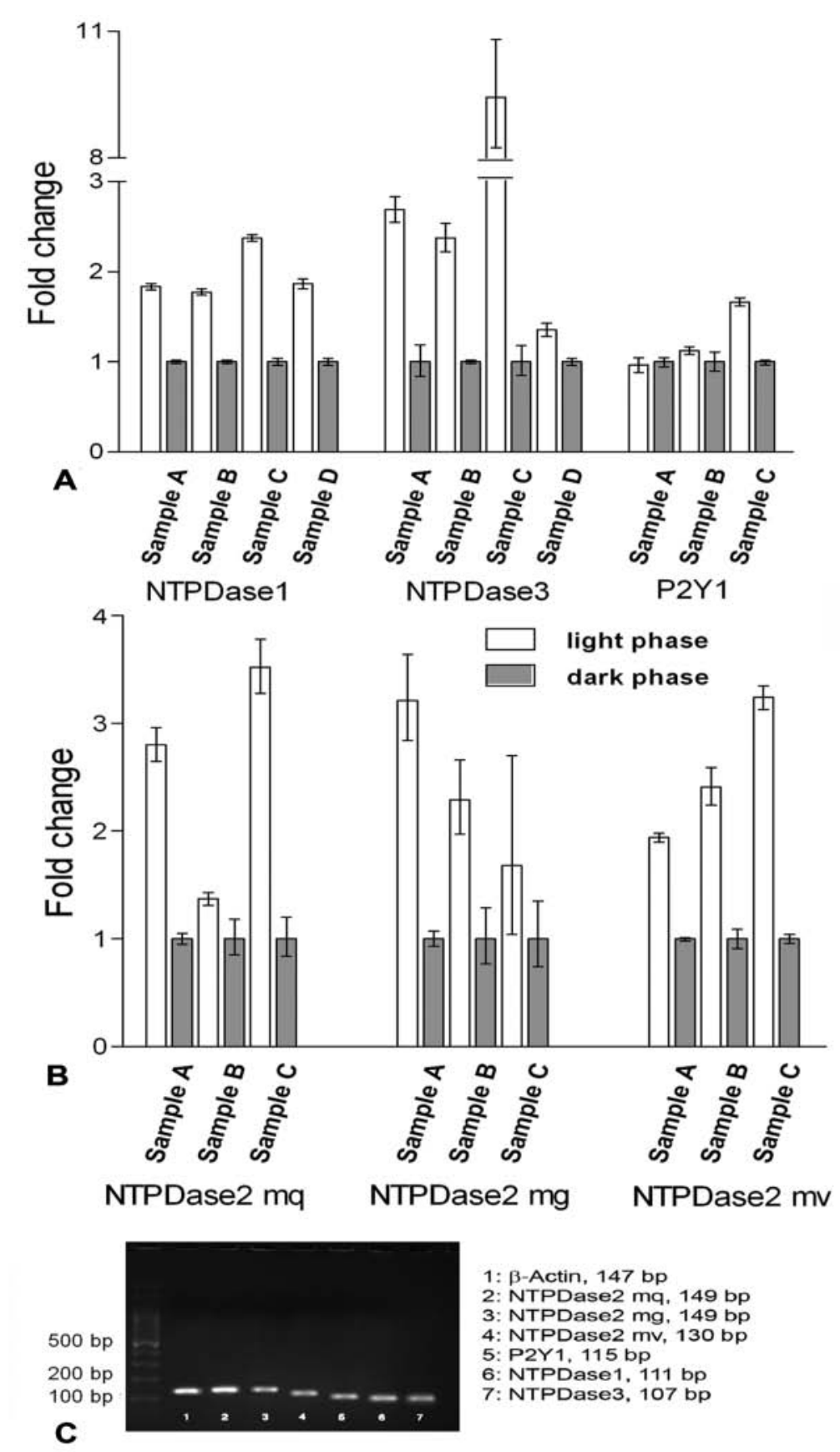

Figure 4. NTPDases and P2Y1 receptor expression in the zebrafish retina identified by real time quantitative RT-PCR (qPCR). RNA was purified from 3-4 pools (samples A-D) of 10 retinas each obtained at the mid-light phase (ZT7-9) or beginning of the dark phase (ZT14-16) of a 14:10-h LD cycle (ZT0 is lights on and ZT14 lights off). Total RNA was treated with DNAse-I, retro-transcribed to cDNA, and amplified by qPCR. One microgram of cDNA, except for NTPDase2 $m g$ isoform $(2 \mu \mathrm{g})$, was used as template for qPCR reactions, with primers for $\beta$-actin (reference) or specific primers for each target gene. Fold change is the media from triplicate values (for each reaction) normalized to an internal reference and a calibrator sample (ZT14-16) that was calculated by the $2^{-\triangle \Delta C T}$ method. Propagated errors were estimated from triplicate SD by using standard methods. Error ranges (brackets) were calculated as $2^{-(\Delta \Lambda C T \pm \text { propagated SD) }}$. Amplified cDNA products were separated by electrophoresis in a $2 \%$ agarose gel (C). NTPDase2 isoforms: $m q, m g, m v$.
eNCT levels and hence by the activity of plasma membrane-bound NTPDases. NTPDase mRNA expression is differentially regulated during the LD cycle.

\section{S-Phase Cell Activity in the CMZ Shows Day-Night Oscillations in LD That Persist for One Day in Constant Darkness}

It has been reported that the proliferative activity of rod precursors in the ONL of a teleost fish retina (Haplochromis burtoni) exhibits circadian driven variations with a peak at night (Chiu et al., 1995). Here we showed day-night variations of S-phase nuclei number during a regular LD cycle that are at least 3 times higher at daylight than in darkness. A further control was made because, as aforementioned, zebrafish for light intervals were maintained in a separate room with an inverted (I) LD phase from zebrafish for night intervals. Results indicated that BrdU-positive nuclei number peak is resynchronized by light to a 12-h shifted LD cycle (as shown in Supplemental Figure S2), and the observed differences between groups at daylight or night intervals are not due to any uncontrolled environmental variable.

Rod precursors in the ONL (Johns and Fernald, 1981) or proliferative cells in the INL (Julian et al., 1998; Battista et al., 2009) were virtually unlabeled under our experimental conditions ( $2 \mathrm{~h}$ of $\mathrm{BrdU}$ exposure); therefore, we could not assess day-night variations in these cell populations. S-phase activity in the CMZ, which involves a different neurogenic cell population, seems to be coincident with a higher behavioral activity (Cahill et al., 1998) and visual sensitivity at daylight (Li and Dowling, 1998). Mitotic division in vertebrates occurs several hours after $S$ phase-in our experiments, at the end of the light phase or beginning of the dark phase. Therefore, BrdU in our experimental conditions labeled S-phase nuclei that did not reach the mitotic phase (Matsuo et al., 2003). Oscillations of S-phase cell 
activity persisted $24 \mathrm{~h}$ in DD, suggesting that they are driven at least in part by a circadian pacemaker (Cahill, 2002; Dekens et al., 2003). Nonetheless, this putative rhythm rapidly dampened and disappeared from the second day on in DD. BrdU-positive nuclei number throughout day 2 in DD was low. In contrast, during day 4 in DD, S-phase cell activity showed a global nonoscillatory increase, suggesting a lightindependent cell cycle regulation to sustain cell proliferation and hence retinal growth. Light does not regulate cell cycle entry instantaneously, because S-phase cell activity showed similar levels the first $2 \mathrm{~h}$ of light (ZT0-2) or in darkness (ZT19-21). Together, these results might indicate that S-phase nuclei entry in the CMZ is driven by a weak circadian oscillator that maintains DD cyclic activity for 1 day. Results also suggest that S-phase cell activity is partially lightdriven, because LD cycle absence causes a delayed suppression of cyclic oscillations on day 2. A combination of a clock-driven and a delayed light-driven extracellular diffusible signal and/or retinal cell activity, probably including photoreceptor or another kind of photosensitive cells (Cheng et al., 2009), might be responsible for increasing daylight S-phase activity in the CMZ.

\section{Apyrase and ARL67156 Exert a Similar Effect on Daily Variations of S-Phase Cell Activity}

Apyrase hydrolyses triphosphate and diphosphate nucleotides and hence terminates their action on P2 receptors. The action of apyrase by producing AMP indirectly favors adenosine formation. However, the lack of effect of 8-SPT suggests that endogenous extracellular adenosine does not regulate S-phase nuclei number in the CMZ. Therefore, the inhibitory effect of apyrase on S-phase activity in the CMZ, in either the light or dark phase, might result from hindering nucleotide action on P2 receptors. In contrast, hexokinase treatment, which hydrolyzes extracellular ATP, did not affect S-phase nuclei number. This might indicate that ATP is not essential for controlling S-phase activity in the CMZ. Another possible candidate is ADP because it binds to specific P receptors-unlike $\mathrm{AMP}$ —and apyrase quickly hydrolyzes it. Hexokinase activity may produce ADP; however, no effect of hexokinase was observed in either light or darkness. ADP produced by hexokinase activity at daytime could be redundant with extracellular ADP produced by other enzymatic reactions. Our data suggest that at night, there is less extracellular ADP; nonetheless, hexokinase was ineffective in producing enough ADP to exert a significant effect on S-phase cell activity. This might be due to a very low extracellular ATP concentration at night. Alternatively, ADP produced within the vitreous by hexokinase could be rapidly hydrolyzed by endogenous ectoATPase activity (Battista et al, 2009).

Like the apyrase effect, ARL67156 significantly diminished S-phase nuclei number in the CMZ by inhibiting extracellular hydrolysis of nucleotides. Therefore, these results also suggest that ATP presence by itself in the extracellular milieu is not sufficient to regulateS-phase entry. Presence of ectoATPase activity mainly driven by NTPDases is crucial for hydrolysis of eNCT and key for S-phase induction in the $\mathrm{CMZ}$ at daylight and night.

\section{P2Y1 Receptors Mediate \\ Extracellular Nucleotide Effect on S-Phase Cell Activity in the CMZ}

A treatment with ADP $\beta S(25 \mu \mathrm{M})$ led to significant increases in BrdU-positive cell number at nighttime. An elevated level of this ADP analogue for $20 \mathrm{~h}$ was a signal sufficient to increase S-phase cell number at night to mid-light values. There is common agreement that P2Y1 receptors have a greater selectivity for ADP than ATP (Burnstock, 2007). In concordance with ADP $\beta$ S results, a selective antagonist for P2Y1 receptors MRS2179 significantly inhibited S-phase cell number mid-light increase. In contrast, antagonists selective for other ADP-activated (P2Y13) or ATP-activated receptors (P2X1/2/3 and P2Y11) were ineffective. These results suggest that the enhanced daylight S-phase cell number is principally mediated by ADP action on P2Y1 metabotropic receptors. Further evidence supporting this finding indicated that ADP is a key signal for injury-induced cell proliferation in the zebrafish retina (Battista et al., 2009). Moreover, we have also demonstrated-for the first time-that P2Y1 receptor mRNA is expressed in the zebrafish retina.

\section{RNA Messengers for Different Plasma Membrane-Bound NTPDases Are Expressed in the Zebrafish Retina and Exhibit Daily Variations}

We have previously found by immunocytochemistry that NTPDases 1 and 2 are differentially localized 
in mature layers as well as in the CMZ of the zebrafish retina (Ricatti et al., 2009). We show here that NTPDase1, NTPDase3, and 3 isoforms of NTPDase2 mRNAs previously described in the brain (Rico et al., 2006; Appelbaum et al., 2007) are also expressed in the zebrafish neural retina. NTPDases are the main regulating system of eNCT concentration (Zimmermann, 2006). NTPDase1 hydrolyzes triphosphate and diphosphate nucleotides with an activity ratio of 1 to 1 . NTPDase2, in contrast, hydrolyzes principally ATP accumulating extracellular ADP, whereas NTPDase3 exhibits an intermediate activity (Kukulski et al., 2005). In this report, we described day-night variations of mRNA levels for all NTPDases examined, except for the $m g$ isoform, which only showed a significant change in 2 of 3 samples and seems to be the less abundantly expressed because, as mentioned before, we had to use twice the amount of cDNA to detect it. NTPDase gene expression at mid-light doubles in average the expression observed after a short time in darkness. Presumably, NTPDase activity might be higher at daylight than at night consistent with a tighter regulation of extracellular nucleotide levels.

\section{Different Levels of eNCT as Well as NTPDase Activity Might Be Critical to Drive CMZ S-Phase Cell Activity During the LD Cycle}

A recent report has described that larval zebrafish electroretinographic activity is virtually null at night and is under circadian control (Emran et al., 2010). The authors suggest that metabolic activity is significantly diminished at night to conserve energy. In the adult zebrafish, a decreased retinal activity at night has also been reported (Li and Dowling, 1998). Presumably, less activity and lowest metabolism might imply a reduced content of extracellular ATP (which depends on intracellular ATP concentration). This fact, in addition to a lower NTPDase activity, might contribute to low levels of extracellular ADP at night. Alternatively, although we did not detect day-night variations of P2Y1 receptor mRNA levels, a decreased response to eNCT at night cannot be ruled out. Therefore, the assumed highest levels of eNCT as well as an increased NTPDase mRNA expression, which might be under circadian control, could induce S-phase cell entry in the CMZ (Martins and Pearson, 2008) at daylight or mid-subjective day for $24 \mathrm{~h}$ in DD. ARL67156, apyrase, hexokinase, and P receptor antagonist treatments indicated that the BrdU-positive nuclei increase chiefly depends on activation of P2Y1 receptors by ADP as well as NTPDase extracellular activity. The low number of S-phase nuclei in the CMZ at night is also regulated by extracellular ADP-probably at much lower concentrations-because exogenous ADP $\beta S$ was able to increase S-phase activity to daylight levels. When eNCT were scavenged or NTPDase activity inhibited, S-phase entry in the CMZ decreased to almost null values. It is tempting to suggest that the very few remaining proliferating cells might be stem cells, whose cell cycle regulation is independent of $\mathrm{eNCT} / \mathrm{S}$.

We have demonstrated that NTPDase mRNA expression levels show day-night variations. These might be driven by a weak endogenous oscillator and / or the photic stimulus to regulate eNCT day-night concentration and hence precursor cell S-phase entry in the CMZ. It remains to be shown, however, if light, a circadian mechanism, or both are indeed capable of regulating eNCT concentration or ATP release by retinal cells.

\section{ACKNOWLEDGMENTS}

We thank Drs. Adriana De Siervi and Luis Gonzalez Flecha for technical assistance and Drs. Pablo J. Schwarzbaum and Daniel P. Cardinali for reading and comments on the manuscript. Grant sponsors: Agencia Nacional de Promoción Científica y Tecnológica (ANPCyT-BID1728/04-PICT2004-N²5422); Consejo Nacional de Investigaciones Científicas y Técnicas (CONICET PIP2004-Nº012). Argentina.

\section{NOTE}

Supplementary online material for this article is available on the Journal of Biological Rhythms website: http://jbr.sagepub.com/supplemental.

\section{REFERENCES}

Appelbaum L, Skariah G, Mourrain P, and Mignot E (2007) Comparative expression of $\mathrm{p} 2 \mathrm{x}$ receptors and ectonucleoside triphosphate diphosphohydrolase 3 in hypocretin and sensory neurons in zebrafish. Brain Res 1174: 66-75.

Battista AG, Ricatti MJ, Pafundo DE, Gautier MA, and Faillace MP (2009) Extracellular ADP regulates lesioninduced in vivo cell proliferation and death in the zebrafish retina. J Neurochem 111:600-613. 
Burnstock G (1978) A basis for distinguishing two types of purinergic receptors. In Cell Membrane Receptors for Drugs and Hormones: A Multidisciplinary Approach, Straub RW and Bolis L, eds, pp 107-118. New York: Raven Press.

Burnstock G (2006) Historical review: ATP as a neurotransmitter. Trends Pharmacol Sci 27:166-176.

Burnstock G (2007) Physiology and pathophysiology of purinergic neurotransmission. Physiol Rev 87:659-797.

Cahill GM (2002) Clock mechanisms in zebrafish. Cell Tissue Res 309:27-34.

Cahill GM and Besharse JC (1995) Circadian rhythmicity in vertebrate retinas: regulation by a photoreceptor oscillator. Prog Retin Eye Res 14:267-291.

Cahill GM, Hurd MW, and Batchelor MM (1998) Circadian rhythmicity in the locomotor activity of larval zebrafish. Neuroreport 9:3445-3449.

Cheng N, Tsunenari T, and Yau KW (2009) Intrinsic light response of retinal horizontal cells of teleosts. Nature 460:899-903.

Chiu JF, Mack AF, and Fernald RD (1995) Daily rhythm of cell proliferation in the teleost retina. Brain Res 673:119-125.

Dekens MP, Santoriello C, Vallone D, Grassi G, Whitmore D, and Foulkes NS (2003) Light regulates the cell cycle in zebrafish. Curr Biol 13:2051-2057.

Emran F, Rihel J, Adolph AR, and Dowling JE (2010) Zebrafish larvae lose vision at night. Proc Natl Acad Sci USA 107:6034-6039.

França GR, Freitas RC, and Ventura AL (2007) ATP-induced proliferation of developing retinal cells: regulation by factors released from postmitotic cells in culture. Int J Dev Neurosci 25:283-291.

Hunsucker SA, Mitchell BS, and Spychala J (2005) The 5 -nucleotidases as regulators of nucleotide and drug metabolism. Pharmacol Ther 107:1-30.

Johns PR and Fernald RD (1981) Genesis of rods in teleost fish retina. Nature 293:141-142.

Julian D, Ennis K, and Korenbrot JI (1998) Birth and fate of proliferative cells in the inner nuclear layer of the mature fish retina. J Comp Neurol 394:271-282.

Kukulski F, Levesque SA, Lavoie EG, Lecka J, Bigonnesse F, Knowles AF, Robson SC, Kirley TL, and Sevigny J (2005) Comparative hydrolysis of P2 receptor agonists by NTPDases 1, 2, 3 and 8. Purinergic Signal 1:193-204.

Li L and Dowling JE (1998) Zebrafish visual sensitivity is regulated by a circadian clock. Vis Neurosci 15:851-857.

Livak KJ and Schmittgen TD (2001) Analysis of relative gene expression data using real-time quantitative PCR and the $2^{-\triangle \triangle C T}$ method. Methods 25:402-408.
Lyall A (1957) The growth of the trout retina. Q J Microsc Sci 98:101-110.

Martins RAP and Pearson RA (2008) Control of cell proliferation by neurotransmitters in the developing vertebrate retina. Brain Res 1192:37-60.

Massé K, Bhamra S, Eason R, Dale N, and Jones EA (2007) Purine-mediated signaling triggers eye development. Nature 449:1058-1062.

Matsuo T, Yamaguchi S, Mitsui S, Emi A, Shimoda F, and Okamura H (2003) Control mechanism of the circadian clock for timing of cell division in vivo. Science 302: 255-259.

Newman EA (2003) Glial cell inhibition of neurons by release of ATP. J Neurosci 23:1659-1666.

Newman EA (2006) A purinergic dialogue between glia and neurons in the retina. Novartis Found Symp 276: 193-202.

Pearson RA, Dale N, Llaudet E, and Mobbs P (2005) ATP released via gap junction hemichannels from the pigment epithelium regulates neural retinal progenitor proliferation. Neuron 46:731-744.

Reh TA and Fischer AJ (2001) Stem cells in the vertebrate retina. Brain Behav Evol 58:296-305.

Ricatti MJ, Alfie LD, Lavoie EG, Sevigny J, Schwarzbaum PJ, and Faillace MP (2009) Immunocytochemical localization of NTPDases1 and 2 in the neural retina of mouse and zebrafish. Synapse 63:291-307.

Rico EP, Rosemberg DB, Senger MR, Arizi M, Bernardi GF, Dias RD, Bogo MR, and Bonan CD (2006) Methanol alters ecto-nucleotidases and acetylcholinesterase in zebrafish brain. Neurotoxicol Teratol 28:489-496.

Robson SC, Sévigny J, and Zimmermann H (2006) The E-NTPDase family of ectonucleotidases: structure function relationships and pathophysiological significance. Purinergic Signal 2:409-430.

Schefe JH, Lehmann KE, Buschmann IR, Unger T, and Funke-Kaiser H (2006) Quantitative real-time RT-PCR data analysis: current concepts and the novel "gene expression's CT difference" formula. J Mol Med 84: 901-910.

Stenkamp DL (2007) Neurogenesis in the fish retina. Int Rev Cytol 259:173-224.

Westfall TD, Kennedy C, and Sneddon P (1997) The ectoATPase inhibitor ARL 67156 enhances parasympathetic neurotransmission in the guinea-pig urinary bladder. Eur J Pharmacol 329:169-173.

Zimmermann H (2006) Ectonucleotidases in the nervous system. Novartis Found Symp 276:113-128. 